\title{
Arborescences
}

Revue d'études françaises

\section{Géocritique du fleuve dans les mémoires de la Commune de Louise Michel}

\section{Valérie Narayana}

Numéro 8, décembre 2018

La Seine littéraire au XIX ${ }^{\mathrm{e}}$ siècle

URI : https://id.erudit.org/iderudit/1055884ar

DOI : https://doi.org/10.7202/1055884ar

Aller au sommaire du numéro

Éditeur(s)

Département d'études françaises, Université de Toronto

ISSN

1925-5357 (numérique)

Découvrir la revue

Citer cet article

Narayana, V. (2018). Géocritique du fleuve dans les mémoires de la Commune de Louise Michel. Arborescences, (8), 64-79. https://doi.org/10.7202/1055884ar
Résumé de l'article

Une approche géocritique examine le motif fluvial dans l'écriture autobiographique de Louise Michel et plus particulièrement dans $L a$ Commune : histoire et souvenirs (1898). La Seine est prise comme principe figuratif ordonnant le courant des foules, les jeux de pouvoir et le sort des insurgés. Le bassin fluvial est parcouru comme espace mémoriel et métaphorique, à la convergence de forces militaires et géopolitiques. Une poétique de la fluidité fait de ce récit intertextuel, tactique et fragmentaire, un lieu où se reflètent les aléas de la politique et les méandres du progrès.
Tous droits réservés ( $C$ Département d'études françaises, Université de Toronto, 2019
Cecument est protégé par la loi sur le droit d'auteur. L'utilisation des services d’Érudit (y compris la reproduction) est assujettie à sa politique d'utilisation que vous pouvez consulter en ligne.

https://apropos.erudit.org/fr/usagers/politique-dutilisation/ 


\section{SOMMAIRE}

1 Nicolas Gauthier, Université de Waterloo

Sébastien Roldan, chercheur indépendant

Introduction

6 Janine Gallant, Université de Moncton

La Seine de Mérimée et ses ressorts dramatiques

17 Silvia Baroni, Université de Bologne

Au seuil de l'enfer: la Seine dans La Comédie humaine

33 Nicolas Gauthier, Université de Waterloo

La Seine du crime et les petits métiers parisiens

46 Sébastien Roldan, chercheur indépendant

Où est la Seine dans les Tableaux parisiens de Baudelaire?

64 Valérie Narayana, Université Mount Allison

Géocritique du fleuve dans les mémoires de la Commune de Louise Michel

80 Peter Vantine, Saint Michael's College

Au bord de la Seine avec les Goncourt

102 Lola Kheyar Stibler, Université Sorbonne Nouvelle

«Hydrargyre» de Maurice de Fleury ou l'imagination de la matière 


\title{
Géocritique du fleuve dans les mémoires de la Commune de Louise Michel
}

\author{
Valérie Narayana, Université Mount Allison
}

\section{Résumé}

Une approche géocritique examine le motif fluvial dans l'écriture autobiographique de Louise Michel et plus particulièrement dans La Commune: histoire et souvenirs (1898). La Seine est prise comme principe figuratif ordonnant le courant des foules, les jeux de pouvoir et le sort des insurgés. Le bassin fluvial est parcouru comme espace mémoriel et métaphorique, à la convergence de forces militaires et géopolitiques. Une poétique de la fluidité fait de ce récit intertextuel, tactique et fragmentaire, un lieu où se reflètent les aléas de la politique et les méandres du progrès.

Dans l'iconographie de la Commune, rien de plus célèbre que l'image de la Seine rougie par le feu. Prosper-Olivier Lissagaray en peint le tableau suivant, connu des partisans comme des adversaires:

Les Tuileries brûlent; la Légion d'honneur, le Conseil d'État [...]. Le flot rouge de la Seine reflète les monuments et double l'incendie. Chassées par un souffle de l'Est, les flammes irritées se dressent contre Versailles et disent au vainqueur de Paris qu'il n'y retrouvera plus sa place et que ces monuments monarchiques n'abriteront plus de monarchie (2000: 334).

De nombreux témoignages attestent l'effet paradoxal du fleuve embrasé ${ }^{1}$. Or, la Seine ne se réduit pas à ce lieu d'immolation finale, du moins pas sous la plume de la communarde Louise Michel. Notre article propose d'examiner, au moyen d'une approche géocritique, le motif fluvial dans l'ouvrage autobiographique que Louise Michel publie en 1898, La Commune: histoire et souvenirs ${ }^{2}$.

L'écrit autobiographique michélien est complexe car plusieurs années séparent les événements rapportés des lourdes conséquences qu'ils auront, dont l'exil en Nouvelle-Calédonie. L'ancienne proscrite doit composer avec des visées difficiles à concilier: d'une part, chanter la gloire de la Commune, d'autre part, expliquer comment un tel échec pourrait se solder en triomphe. Dans son récit entrecoupé de prolepses et d'analepses, l'image du fleuve relie le passé et le futur. La Seine, dans ses manifestations textuelles, documente la dissolution de l'Empire et de l'identité nationale. Quand le récit michélien représente le fleuve, c'est en milieu parisien; mais il y est également question des remparts naturels que forment les deux massifs de collines qui bordent le fleuve dans l'actuel département des Hauts-de-Seine, et des affluents que sont les canaux du Nord-Est. Ainsi, de Sarrebruck à la Semaine Sanglante, le réseau fluvial - écrin de l'agora, tranchée ou chenal - s’impose comme un haut lieu de la mémoire communarde.

1. La collection Les Reporters de l'histoire, recensement thématique de témoignages d'écrivains, est ici utile; le numéro sur la Commune montre l'omniprésence des allusions à l'incendie (Priollaud 1983).

2. Nous désignerons désormais cet ouvrage par le seul nom «Commune». 
L'écriture michélienne est solidaire. Les exploits et témoignages de plusieurs camarades y trouvent leur place et s'étendent sur plusieurs pages. S'intercalent chansons et poésies. Il en résulte une écriture fragmentaire, où les événements clés doivent être restitués au gré de causalités proposées par une Histoire elle aussi parcellaire, car redevable aux récits de survivants dispersés. Pour restaurer ce contexte, il convient de mettre le texte de Michel en lien avec d'autres écrits, soit communards (ceux de Prosper-Olivier Lissagaray ou de Jules Vallès), soit adverses (ceux de Maxime Du Camp). À cette plurivocité s'ajoutent les multiples facettes d'une Seine appréhendée dans l'ordre physique et symbolique. C'est afin de rendre compte de ces éléments manifestes sur plusieurs plans contigus (historique, sociologique, stratégique) que ce travail recourt à la géocritique.

\section{Seine d'une fin d'Empire: courants géocritiques et axes d'analyse}

Il serait vain de s'en tenir au simple fait chronologique pour décrire l'affrontement de 1870-1871. Il se déploie sur un terrain autant figuratif que littéral; d'abord, en un point focal européen, mais aussi au sein d'un ensemble de savoirs militaires, politiques et techniques, qui lient, tout en les départageant, diverses couches sociales. À cet égard, trois éléments de la théorie géocritique proposée par Bertrand Westphal facilitent l'analyse d'une guerre civile. En premier lieu, il y a ce que l'auteur nomme une «vision stratigraphique» (2007: 222). Grâce à celle-ci, «la représentation d'un espace se révèle intéressante à la croisée de points de vue hétérogènes" car la "déterritorialisation assouplit la rigidité des repères traditionnels» (2007: 223). Cette optique éclaire un deuxième élément: la "transgressivité», état manifeste quand "le territoire cesse d'être univoque», en proie à une "prolifération des centres focaux et une oscillation globale du système de référence» (2007: 89):

Dès lors, le territoire s'occulte au profit d'une territorialité évolutive, toute tentative de délimitation étant vouée à l'éphémère. Pour sa part, la déterritorialisation est absolue lorsqu'elle mène au nouveau; elle est relative lorsqu' elle finit par renouer avec la tradition, quoique le territoire, comme les eaux du fleuve d'Héraclite, jamais n'aura deux fois la même nature (2007: 89).

En troisième lieu, la plurivocité admise par la géocritique permet un décloisonnement générique. En réplique à la question : "à partir de quel moment un ouvrage est-il un ouvrage de fiction?", Westphal note que son approche "se fonde sur un postulat inverse: les espaces humains ne deviennent pas imaginaires en intégrant la littérature; c’est la littérature qui leur octroie une dimension imaginaire, ou mieux: qui traduit leur dimension imaginaire intrinsèque en les introduisant dans un réseau intertextuel» (2000: 21). Les notions de "transgressivité » et de "déterritorialisation" sont propices à l'examen d'une insurrection. L'investissement imaginaire, pour sa part, est essentiel dans l'analyse d'une autobiographie. Quant à la "vision stratigraphique», elle s'applique au texte du retour d'exil, car chez Michel l'ailleurs se greffe à l'antan. Aussi quelques précisions aideront-elles à ordonner les choses.

Les années 1870-1871 recouvrent plus d'un conflit. La guerre déclarée par Napoléon III contre la Prusse en provoque une deuxième, civile. En même temps, l'internationalisation des forces ouvrières est à ses débuts. De là, diverses "strates" d'investissement idéologique face aux incidents. Ainsi, de grands déplacements matériels et symboliques (notamment la proclamation de l'Empire allemand dans la galerie des Glaces de Versailles, suivie par la fuite du gouvernement de la république vers le palais) donnent lieu à une gamme d'interprétations, plus ou moins transgressives. Il convient donc de parler de «déterritorialisations ", au pluriel. À l'enjeu de l'intériorité-extériorité nationale (redéfinie au gré de 
fronts et frontières changeant lors d'opérations militaires), il faut superposer l'involution créée par la Commune, où l'ennemi perçu par Versailles investit la Cité de l'intérieur.

Cet enchevêtrement d'intérêts est évoqué par Michel. Les incidents déterminants dont elle témoigne se trouvent associés au fleuve qui définit divers espaces démographiques tout en structurant leurs mouvements stratégiques. En région parisienne, ces mouvements se déploient généralement le long d'un axe nord-est/sud-ouest. À l'approche des Prussiens succède, en direction inverse, non pas une résistance dirigée vers l'étranger mais bien le massacre de la Semaine Sanglante, qui débute à la Porte de Saint-Cloud, située au sud-ouest de Paris, pour aboutir au cimetière du Père-Lachaise, dans le nordest de la ville. À cette violence, Michel oppose le parcours de Gustave Flourens, figure romantique combattant dans les hauts de la Seine. Le destin du général communard est cependant tragique. Pour pallier cette déception, Michel rescape la mémoire de ses camarades avec une poétique qu'on pourrait dire phréatique: leur sang versé dans la Seine prépare le limon pour le combat futur.

Cette image, d'ailleurs assez convenue dans l'iconographie des insurgés, côtoie une vision sociale plus complexe chez Louise Michel, qui condamne avant tout l'hypocrisie et la lâcheté. Bien que méfiante à l'endroit des hiérarchies, elle apprécie l'initiative et saura gré, lors du siège de Paris, à la débrouillardise du bâtisseur, patron ou ouvrier. Son matérialisme anticlérical lui fait valoriser les fruits de l'inventivité technique, créés par l'entrepreneur et partagés par ses travailleurs. Le Paris qu'elle décrit est divisé par des cours d'eau qui reflètent ces fonctions sociales. Pour comprendre ces divisions, il est utile de se référer aux propos d'Henri Lefebvre, qui, dans ses travaux sur la Commune, manifeste de l'intérêt pour la géographie urbaine, optique qui inspirera son célèbre essai de 1974, La Production de l'espace. L'historien souligne l'emprise des réalités sociologiques sur la configuration de l'urbanité, tant concrète que symbolique. Il y explique comment le serment bourgeois, remontant à l'époque d'Étienne Marcel, comporte une dimension à la fois commerciale et tribale, du fait que l'inventivité technique assure un certain ascendant sur le fait matériel (1965: 145-148). La fidélité y est donc indissociable de l'autonomie. Ce paradoxe est incarné par la milice qu'est la garde nationale, dont les généraux sont souvent patrons et petits industriels.

En 1871, la loyauté des miliciens est éprouvée par les défaites militaires. L'armée régulière est elle aussi polarisée. Certains soldats restent fidèles à Versailles mais d'autres se rallient aux insurgés. Ce choix est intensifié par une nouvelle enchère: le prestige des francs-tireurs. Admirés et revenus glorieux des guerres de liberté, ils épousent la cause communarde. Ces héros tirailleurs se battront dans les hauts de la Seine, flanqués par les miliciens parisiens du Nord-Est, région dont la richesse aquifère la lie au réseau fluvial urbain par la longue évolution d'une technè mercantile.

Ce sont tous ces intérêts que la géocritique recense; mais il importe d'abord de situer les choses à l'aide d'une mise en contexte échelonnée en trois temps: d'abord, un résumé des événements menant à la chute de l'Empire et à l'avènement de la République; ensuite, un survol de la situation de la garde nationale à l'époque; enfin, un aperçu de l'évolution urbaine à la veille du conflit. Les propos de Michel nécessitent une telle contextualisation. De plus, ces enjeux peuvent être rattachés à divers espaces fluviaux. Ceux-ci seront abordés d'ouest en est. En effet, ce n'est qu'en tenant compte de la dualité de la Seine (mêlant l'abstraction de la frontière départementale au fleuve qui la traverse) que le recoupement de deux conceptions du pouvoir - l'une tribale, l'autre délibérative - peut être envisagé. Or, c'est une telle «stratigraphie» de l'espace fluvial qui ressort du texte de Michel. 


\section{Seine de la République: les avals d'une déterritorialisation (Montretout, Saint-Cloud, Versailles)}

Suivant l'axe évoqué plus tôt, la première région fluviale à considérer est celle traversant les hauts de la Seine. La guerre y est proclamée le 19 juillet 1870 à Saint-Cloud, où le palais impérial est bombardé trois mois plus tard. Les obus font couler beaucoup d'encre, leur origine étant peut-être française (Vachon 1880: 2). La garde nationale de la capitale soutient initialement l'armée mais au cours des onze mois qui suivent, les échecs éprouvent la loyauté de la milice et l'amènent à privilégier ses propres moyens de défense. La débâcle de Montretout (au nord de Saint-Cloud) en janvier 1871 se solde par l'affaire des canons, cause d'un retranchement vers les Buttes en février. En effet, Alphonse Thiers ayant trahi la milice en convoitant son arsenal privé, la garde lui préfèrera l'audace communarde et la verve de tribuns comme Henri Rochefort (souvent cité par Michel). Sur le plan géocritique, ce retrait vers les régions canalisées du Nord-Est évoque le rapport guerrier et mercantile liant la bourgeoisie à la Cité: le serment est indissociable d'une souveraineté concrète. Mue autant par une éthique distributive qu'une mystique tribale, la milice va défier Thiers, qui fuira à Versailles à la mi-mars.

La guerre déclarée huit mois plus tôt est désastreuse: Napoléon III gagne à Sarrebruck puis accumule les défaites. L'empereur est fait prisonnier. La nouvelle parvient à Paris le 2 septembre et la République est proclamée le 4. Or, le fait républicain s'envisage de façon très variable chez les Parisiens ${ }^{3}$. Un gouvernement de défense nationale est formé avec entre autres les trois Jules (Favre, Simon, Ferry), Léon Gambetta et Henri Rochefort (tiré de prison après l'affaire Victor Noir ${ }^{4}$ ). Entretemps, les Prussiens avancent; vers la mi-septembre, Favre rencontre Bismarck, qui instaure un blocus le 19. Raillant ce qu'il voit comme la "clique socialiste» régnante, le chancelier menace de ramener l'empereur à Paris en fourgon (Rougerie 2014: 22). Favre décide alors de poursuivre le combat pour ne pas avoir à céder l'Alsace-Lorraine et payer cinq milliards de francs d'indemnité.

Les "rouges", dès l'annonce de la captivité de Napoléon III, forment des comités de vigilance dans les arrondissements sympathisants. Ils s'unissent sous la bannière du Comité central de Défense nationale, conçu pour signaler aux dirigeants la volonté des assiégés. L’on désignera ce groupe comme le Comité de la Corderie, en référence au lieu de sa réunion inaugurale (Lefebvre 1965: 184-185). L'appellation permet de le distinguer d'un autre, le Comité central, qui fédère un mois plus tard les forces républicaines de la garde, aux croyances plus diverses (Azéma et Winock 1970 : 48-49).

Ces patriotes veulent défendre Paris. Le général Trochu organise donc la sortie de Montretout en janvier. Michel décrit ainsi la rive ouest du fleuve juste au nord des ruines du pont de Sèvres: «Là restèrent par centaines [...] des gardes nationaux, hommes du peuple, artistes, jeunes gens; la terre but le sang de cette première hécatombe parisienne, elle en devait être saturée» (Commune: 90). La milice avait été envoyée sous Rochebrune, colonel décoré de la Légion d'honneur pour ses exploits. Comme plusieurs militaires issus de milieux indigents, il se distingue lors de campagnes éprouvantes. Favorable à la

3. Rougerie explique que, dès le 3 septembre, plusieurs «révolutionnaires purs» espèrent être ovationnés par la foule assemblée à l'Hôtel de Ville. Elle leur préfère le gouvernement provisoire présidé par Trochu, militaire breton orléaniste. Cette concession a été faite à la réaction (2014: 19-20).

4. L'écrivain et journaliste, au sortir d'un dîner avec George Sand en février 1870, se fait arrêter. Rochefort avait dénoncé l'assassinat par Pierre Bonaparte de Victor Noir. (Ce journaliste avait été envoyé chez le neveu de Louis-Napoléon par un directeur de journal corse qui avait maille à partir avec le belliqueux parent de l'empereur; le jeune homme avait été abattu sommairement, car on l'avait pris pour un témoin de Rochefort que Bonaparte voulait confronter en duel.) Révolté d'être pris comme prétexte de ce meurtre, le pamphlétaire insulte la famille du coupable et est accusé d'outrage à l'empereur. Le procès acquitte Pierre Bonaparte (Roubaud 1954: 59-63). 
Commune, zouave intrépide, il est estimé. Son $19^{\mathrm{e}}$ régiment accueille aussi Amilcare Cipriani, garibaldien cité longuement par Michel. Ce dernier explique comment la garde se trouva exposée aux feux des soldats prussiens et français. Ce troublant détail accrédite la thèse selon laquelle, aux yeux du gouvernement «modéré» du 4 septembre, un pacte avec l'ennemi étranger aurait été préférable à une Commune populaire protégée par les miliciens (Marx 1963) : ceux-ci «étaient mitraillés en face par les Prussiens, derrière par le Mont-Valérien qui envoyait ses obus sur nous, croyant viser l'armée ennemie» et «sur la droite, nous étions mitraillés [par] une batterie française [qui] trouvait le moyen de nous prendre pour les Prussiens» (Commune: 91). Cipriani survivra mais Rochebrune succombera (Commune: 86-91).

Par le biais du récit de son ami, Michel documente ces tirs français atteignant la milice. Son autobiographie suggère aussi que le général Trochu (nommé par le gouvernement modéré), se méfie de la garde et la traite avec ambivalence. S'il mise sur la popularité de Rochebrune (admiré par les miliciens), il néglige les conseils de celui-ci. Le célèbre tirailleur, comprenant l'esprit de corps, désapprouve le plan de Trochu qui fractionne la garde en la mettant parmi les soldats. Rochebrune avait aussi critiqué la tactique déployée sur le haut-lieu stratégique qu'était la rive ouest du fleuve (Commune: 86-91; Lefebvre 1965: 193-195). La milice décimée gardera souvenir du mépris de Trochu envers le savoirfaire du héros tombé à Montretout. Au carcan de l'armée régulière, certains miliciens finiront par préférer l'autonomie des francs-tireurs dont l'agilité séduit. Il y a lieu d'être frappé par la modernité du rapport entre ces héros d'origine souvent modeste (bon nombre sont fils d'immigrants ou étrangers) et le patriote de banlieue ému par leur prouesse.

Il faut souligner le potentiel transgressif de ces agents. Le pouvoir en dispose dans les hauts de la Seine - là où le fleuve sépare, tout en les reliant, Paris et Saint-Cloud. Ce lien n'est pas que physique: c'est dans la capitale que se gèrent les enjeux affectant la garde, comme l'affaire des canons, qui entraîne la «fuite à Versailles» de Thiers. Cette déterritorialisation est précédée de manifestations répétées réclamant la République et tenues au bord de la Seine. En effet, au sein du projet de défense nationale se trouve le cœur de la question citoyenne: celle du vote, à l'ère du suffrage censitaire. L'enjeu du cens aide à comprendre le retranchement de la milice offusquée et il importe de l'aborder en amont des hauts, soit à Paris. Si, pour conter les rives de l'ouest, Michel prête la plume à Cipriani, c'est elle qui témoigne des événements sur la grève de la Cité. Ponts et parvis urbains lui inspirent une poétique fluviale qui influence ses images: de phréatiques, elles deviennent houleuses.

\section{Scènes de la vie de milice: flux et reflux de la Garde (La Concorde, la Chambre, I'Hôtel de Ville)}

En remontant le fleuve d'ouest en est, la Concorde, la Chambre et l'Hôtel de Ville dominent la Seine. C'est entre ces lieux que se déroulent les principaux événements menant à l'établissement de la Commune. Michel narre les moments forts où l'on se réunit au cœur de la ville: le 4 septembre (l'effondrement de l'Empire), le 31 octobre (la promesse - tôt brisée - d'instituer la Commune), le 22 janvier (la libération de Flourens), le $1^{\text {er }}$ mars (l'entrée des Prussiens dans Paris).

Alors que pour la III ${ }^{\mathrm{e}}$ République, la Seine de 1871 est le miroir des Tuileries embrasées, pour la Communarde, le fleuve symbolise un lieu plus fugitif, bordant une agora illusoire. Dans le contexte de la guerre civile, il est un lieu tactique, un conduit vers le cour de la ville; mais il est aussi un début d'enceinte autour de celle-ci. Sa berge, bornant l'hôtel de ville, "crée» en quelque sorte la foule qui doit s'y concentrer. En cela, le fleuve détermine d'autres courants, métaphoriques, notamment ceux des Parisiens assemblés et surveillés par la garde qui entraîne ou rive l'opinion. 
Écrivant pour la postérité, Michel se doit de ressusciter la foule. Aussi recourt-elle à des tropes plutôt convenus pour le faire, comme celui de l'eau mouvante: l'assemblée est tantôt torrent, tantôt mer. Or, pour la militante, le lieu d'assemblement est stratigraphique car ce souvenir se mêle à celui de l'exil. De là, des échappées plus abstraites, évoquant le large - et, dans la poétique de l'insurgée, ce qui singularise la fluidité de la foule doit être mis en rapport avec sa vision de la milice.

La Commune de Michel décrit un corps social parisien qui est avant tout courant, traversé de forces, aux sens propre (voire militaire) comme figuré. Le 4 septembre, une "foule houleuse» afflue, "tout le jour, la nuit, elle augment[e] encore» (Commune: 69). Puis, "vers midi arrivèrent [...] des gardes nationaux armés", et «[d]evant eux, les municipaux sabre au clair [...] se replièrent avec les sergents de ville quand les gardes nationaux s'avancèrent baïonnette au canon" (Commune: 70-71). Les forces dépeintes (milices municipale et nationale, sergents) semblent miscibles. Le 31 octobre,

[c] eux qui, le 4 septembre, s'étaient dirigés vers la Chambre allèrent vers l'Hôtel de Ville; parfois sur le chemin, on rencontrait quelque troupeau moutonnier, racontant que l'armée prussienne avait manqué être coupée en deux ou trois tronçons, je ne sais plus par qui; ou bien déplorant que les officiers français n'eussent pas connu un petit chemin qui les eût menés droit au cœur de l'ennemi; d'autres encore ajoutaient: Nous tenons toutes les routes. — Les trois tronçons c'étaient trois armées allemandes et c'étaient elles [sic] qui tenaient toutes les routes (Commune: 82).

Aussi l'affluence ballotte-t-elle des épaves: «Les courants suivaient la marche vers l'Hôtel de Ville. Venant de tous les côtés, on bousculait les gobeurs et les mouchards, la mer humaine grossissait» (Commune: 83). Le secours de la milice porté en septembre peut donc alimenter la bêtise et la dénégation tribales pressenties en octobre; la "transgressivité » éventuelle de la garde ne rassure pas forcément Michel, qui préfère la solidarité ouvrière à un nationalisme résorbant gauche et droite. L'attitude michélienne face à la garde est liée au rapport équivoque que cette force entretient avec le pouvoir.

Créée à la demande des Jacobins en 1789, la milice sert divers maîtres au gré des régimes. Elle défend (en principe) son propre territoire, laissant le gain frontalier à l'armée constituée (Forrest 2006: par. $5^{5}$ ). La garde survit, sous plusieurs formes, jusqu'à la fin de la Commune (Forrest 2006; Larrère 2007). Elle est citoyenne (en principe, encore une fois) : le cens attire en ses rangs les plus nantis, d'où le mépris populaire à son égard si elle tolère l'abus de pouvoir (Michelet 1888; Dansette: 49-526).

D'où, aussi, chez les dirigeants, une diversité d'attitudes envers la milice (dont l'image favorable mais précaire de 1792 perdure) (Forrest 2006; Larrère 2007). Napoléon s'en sert avec prudence; Charles X la trouve menaçante et la dissout, au péril de son prestige militaire; Louis-Philippe la convoite en l'écartant au besoin. En 1839-1840, lors de la réforme électorale, les gardes organisent des banquets pour obtenir le droit de vote. Larrère explique: "puisque le régime reconnaît aux gardes la capacité de le défendre par les armes, comment leur refuser la capacité à élire les députés?» (2007: par. 33). Le vote ne s'ensuit pas mais la garde a fait sentir sa présence (Forrest 2006: par. 13). L'enjeu de la représentativité, cher à Michel, y est désormais lié. La dualité idéologique de la garde trouve divers échos politiques car la dimension sacrificielle de son service lui vaut une place de choix dans l'argumentaire doctrinaire. La gauche y voit le fils du héros originel de 1792 (Shafer 2005: 44). Quant à la droite,

5. Le soldat et le garde diffèrent du garde mobile recruté en période de crise chez les hommes valides ayant évité jusquelà la conscription par tirage au sort. Le «moblot» épargné a tiré le bon numéro (Guérin 1965: 20; Forrest 2006: par. 24).

6. Michelet dans son Histoire de la Révolution idéalise et exalte l'honneur du service "volontaire», passant sous silence le fait qu'il est souvent «choisi» de préférence à l'indigence (1888: 425-470). 
elle est moins heurtée par le patriotisme du volontaire assermenté (tout bourgeois fût-il) que par la conscription par tirage au sort avec la possibilité d'acheter un remplacement (Bonald condamne cette pratique, la désignant de "traite des Blancs») (Viviani 1901: 122). Les vertus de gratuité et de souveraineté incarnées par la milice (dans ses représentations optimales) sont prisées (quoique différemment) par la droite comme par la gauche, ce qui l'amène à se défier de tractations centristes.

Ainsi, le 4 septembre, la garde célèbre la République; mais les tenants d'un républicanisme de gauche plus averti voient autrement la passation des pouvoirs. Selon eux, ces nouveaux dirigeants n'entendent pas se débarrasser du suffrage censitaire au profit d'un vrai vote populaire. Par ailleurs, en dépit de divergences, les «Rouges» déplorent une interdiction électorale aggravée par l'Empire: Paris, déjà privé de mairie, doit accepter que l'empereur nomme ses maires d'arrondissements et ses adjoints ${ }^{7}$. Ce ferment de sédition fait mieux comprendre le jeu du gouvernement provisoire. Craignant l'émeute, il pèse deux menaces: l'une étrangère, l'autre civile. Pendant deux mois, il évoque la République sans grand détail. Il confirme ainsi la chute de l'Empire et entretient la bonne volonté initiale de la gauche. Il ne change pas pour autant les structures électorales et dit combattre l'ennemi tout en taisant l'imminente reddition de Metz à Bismarck (Commune: 78-79; Lefebvre 1965: 174). Quand la vérité éclate, fin octobre, les Parisiens demandent des comptes. Flourens et Blanqui s'emparent de l'Hôtel de Ville. Captifs, Favre et Simon promettent un plébiscite pour que les représentants soient désormais élus. Ils assurent qu'il n'y aura pas de représailles, mais ne tiennent pas parole car Flourens sera emprisonné (Commune: 84-95; Rougerie 2014: $28^{8}$ ).

Le vote du 5 novembre voit les quartiers ouvriers élire des rouges, dont plusieurs miliciens. Ceux-ci sont souvent d'anciens jacobins, négociants ou patrons d'industrie locale, las d'une productivité ouvrière minée par l'indigence liée à l'agiotage. Leur autorité naturelle se devine lors de la marche de l'ennemi sur l'Élysée. Il faut préciser ce que le Comité Central avait su imposer aux Jules: la Seine départagerait l'espace à surveiller et la milice se chargerait de la rive droite. La garde obtint qu'aucune violence n'éclate. Michel note: "L'armée se retira sur la rive gauche, la garde nationale seule, sans trouble, sans provocation, sans faiblesse, exécuta son programme»(Commune: 113-114).

\section{Scènes de la vie fédérée: une crue de transgressivité (Mazas, Belleville, La Villette)}

Pour bien saisir l'ascendant qu'exercent les généraux fédérés, il faut remonter la Seine juste à l'est du cœur de Paris, où les écluses du bassin de l'Arsenal permettent d'aller à contre-courant. Il importe aussi de remonter dans le temps, au mois précédant la marche prussienne sur la Concorde.

Les tragédies de Montretout et de Buzenval, sorties effectuées le 19 janvier, ont décimé l'armée et la garde. À Paris, les manifestations sont réprimées par balles et par les mêmes généraux associés aux échecs de guerre. Michel raille un général arrivé à Buzenval deux heures plus tard que prévu car

7. Comme l'explique l'historien Merriman, contrairement à tous les autres 36000 villes, villages et hameaux français, Paris n'avait pas le droit d'élire un maire. Le poste avait été aboli en 1794 et encore en juillet 1848. Sous l'Empire, les Parisiens ne pouvaient même pas élire des conseils municipaux d'arrondissement car ceux-ci devaient être nommés par Napoléon (2014: 13-14).

8. Le texte de Lefebvre (dont est largement tiré ce résumé) note que le gouvernement espérait depuis quelque temps se rendre légitime par plébiscite, outil de prédilection de l'Empire. Favre se tire d'affaire en gardant la mainmise et en raflant aux militants l'enjeu électoral. De fait, si les maires d'arrondissement sont enfin élus, le maire de Paris, lui, sera nommé - Ferry, en l'occurrence (1965 : 174-176). 
incapable de traverser la Seine (il est pris en voiture sur le pont de Neuilly): "Ducrot pour une fois n'était pas en retard, aussi il s'agissait de la foule» (Commune: 88 ). Or, ce militaire s'était lui-même déjà plaint de son général en chef, dont l'action à Choisy aux abords de la Seine (en novembre) ne s'était pas non plus produite à son heure. Ce supérieur, nommé Vinoy, bien qu'ayant de fortes ressources à sa disposition - dont des chaloupes canonnières - «ignorait absolument ce qui se passait à côté de lui » (Rousset: 268). Les Communards n'ont donc pas le monopole des dissensions.

Selon Cipriani, cité dans La Commune, "[1]a nouvelle de la défaite de Montretout avait agité les Parisiens à un tel point que le vaillant Trochu n'osa plus s'y montrer; Vinoy prit sa place” " (92). Il écrit: "Pendant tout le temps que dura la bataille de Montretout, je vis Ducrot caché derrière un mur, un prêtre à son côté, et devant eux étendu à leurs pieds un nègre qui avait eu la tête emportée par un obus du Mont-Valérien” "(Commune: 92). Les tirs du mont étant français, cette sinistre trinité brossée par le franc-tireur garibaldien est particulièrement grotesque - et Michel de rappeler que Ducrot, ayant promis de revenir «mort ou victorieux», «ne fut ni l'un ni l'autre» (Commune: 64).

Elle note: «[c]eux qui si longtemps avaient soutenu que le gouvernement ne se rendrait jamais » et «que pas un pouce du territoire» ne serait livré «virent qu'on les avait trompés» (Commune: 100). Cette période clé multiplie les points focaux du siège en causant un retranchement: la milice dès lors méfiante décentralise son arsenal. «Le 27 février, le bruit se répandit dans Paris de l'entrée de l'armée allemande. Aussitôt les Champs-Élysées furent couverts de gardes nationaux»:

On se ressouvint qu'à la place Wagram il y avait des canons que les gardes nationaux des faubourgs avaient achetés par souscriptions, et qui leur appartenaient, pour la défense de Paris [...] chaque quartier avait les siens. Hommes, femmes, enfants s'attelèrent; les pièces de Montmartre [...] sont montées jusqu’à la Butte. Belleville et La Villette traînent les leurs aux Buttes Chaumont (Commune: 112-113).

La géocritique permet de situer cette transgressivité où se manifestent les fondements idéologiques et matériels de la Commune bourgeoise: la Cité est effectivement née d'un serment consacrant autarcie et apport technique. Les Buttes sont liées à la Seine de longue date, car la canalisation urbaine (permettant aux eaux de l'Ourcq d'approvisionner Paris) prolonge une tradition. Les eaux de Belleville avaient longtemps alimenté les fontaines de la ville avec des aqueducs plus ou moins entretenus, conduits de fortune reflétant les rapports entre régions mitoyennes. Les travaux de l'Empire mettent à profit les hauteurs de la localité et y installent un réservoir (Troche 1864: 22). Vers 1860, cet espace encore autonome, traversé de moulins et de vergers, est annexé à la capitale. Élargi par un pan de bourgades ouvrières, Paris se trouve polarisé sociologiquement (Guérin 1966: 10; Montel 2001). À La Villette et Belleville, les ouvriers ont fui la cherté de loyers occasionnée par le plan d'Haussmann et la garde est le «lieu par excellence de sociabilité populaire» (Rougerie: 46). Dans les régions des canaux du nord-est, la représentativité devient un enjeu au cours du siège.

Il est possible de se faire une idée de l'évolution des sentiments parisiens à cet égard en prenant comme balises deux incidents. Il s'agit de l'Affaire de la Villette et de la descente sur la prison Mazas, située à deux pas du quai de la Rapée. Le 14 août 1870, la guerre tout juste déclarée, des Blanquistes tentent de dévaliser l'arsenal d'une caserne de pompiers située sur le bassin de La Villette. Michel narre ainsi la scène, qui se déroule dans un quartier ouvrier:

Un pompier, a-t-on dit, avait été tué; il n'était que blessé et l'a fait connaître lui-même depuis. Le poste était nombreux, bien armé. La police, prévenue on ne sait comment, tomba sur les 
révolutionnaires. Ceux de Montmartre, arrivés tard, virent sur le boulevard désert [la] voiture dans laquelle avaient été jetés Eudes et Brideau, prisonniers, entourée de mouchards et d'imbéciles qui criaient: aux Prussiens! (La Commune: 60-61).

Or, le 22 janvier (trois jours après la défaite de Montretout en hauts de Seine), lors d'une grande manifestation à l'Hôtel de Ville, Cipriani et ses hommes se dirigent vers le Bassin de l'Arsenal tout près de Mazas. Ils en libèrent Flourens, chef des tirailleurs de Belleville qui, depuis sa prise de l'Hôtel de Ville en octobre, a été accusé d'insubordination selon le Figaro (9 décembre 1870: 2). Cette descente est vraisemblablement effectuée au su d'une garde complaisante, indice de transgressivité 9 .

Voilà qui fait mieux comprendre l'affaire des canons du 18 mars ${ }^{10}$. Le $1^{\text {er }}$ mars, l'étranger marche sur Paris, humiliant la capitale qui n’a jamais abdiqué. Le ressentiment culmine quand Thiers décide, le 18 mars, de s'approprier les canons de la garde. Le général Lecomte entraîne donc vers Montmartre ses soldats (tout juste rentrés depuis l'armistice-capitulation, affamés et épuisés). Leur mission doit se faire sous le couvert de la nuit mais ils sont aperçus. La milice accourt et, avec elle, les femmes, dont Michel. L'historiographie communarde chante l'audace féminine dans ce qui suit (Gullickson 1996: 24-56; Eichner 2004: 23; Commune: 129) : les ouvrières grondent les soldats, leur offrent du vin... et ils lèvent la crosse. On les acclame. Cette solidarité se propage dans la ville: les soldats repliés, la milice reprend ses canons. Thiers traverse la Seine et fuit à Versailles.

La Commune est proclamée le 28 mars. Deux mois plus tard, les Versaillais percent à la porte de Saint-Cloud. Du 23 au 28 mai, les fédérés résistent. À la fin de la Semaine sanglante, la Seine incendiée bloque le nord de Paris. Alors, filant le long du fleuve embrasé en chaloupe canonnière, l'armée perce à nouveau. Elle finit d'abattre 25000 insurgés (Azéma et Winock: 165).

\section{Scènes de la vie communarde: courants d'une esthétique insurrectionnelle}

Les images de la Seine chez Michel sont soit convenues soit curieuses, singularité qui s'explique sans doute par l'ambiguïté de son rapport à l'ordre représenté par la milice. Elle partage avec cette dernière des intérêts pour le délibératif mais sans adhérer au tribalisme. Si elle sait rivaliser d'énergie avec la garde (comme en témoigne sa résilience en exil), son influence auprès du démuni dépend moins d'un prestige idéologique que d'un atout plus fuyant: son charisme insolite. Aussi son imaginaire fluvial puise-t-il dans deux éléments: une reconnaissance du populisme propre à l'iconographie communarde et une reconnaissance de sa propre popularité de communarde iconoclaste.

Figure d'exception (néanmoins solidaire), Michel inclut dans son récit (camaraderie oblige) des images de la Seine recyclant le sang versé pour les générations futures (Commune: 90, 152). Cela dit, elle s'écarte de l'idée de continuité transmise par le symbole fluvial. Il semble que l'imaginaire de la proscrite - de celle qui préféra le militantisme à la maternité et dont le singulier ascendant crée des ruptures au sein de catégories établies - ne s'accommode pas toujours de la permanence associée aux aspects directionnels et phréatiques du fleuve. Les images qu'elle propose ne sauraient se borner à une

9. De plus, le 26 février, au cérémonial pour l'anniversaire de la $\mathrm{II}^{\mathrm{e}}$ République, où se trouve la moitié de la garde, le mouchard Vincenzini est «jeté à la Seine par la foule, immolé comme en un massacre rituel pour avoir profané par sa présence un espace sacré» (Lissagaray: 425; Rougerie: 45).

10. Le résumé qui suit est tiré de la description d'Azéma et Winock (1970: 32-36), ainsi que du texte Note sur le concours apporté par la marine pour la répression de l'insurrection de Paris (1871: 2-12). 
analogie pérennisant le fil des choses et réunissant, tout en les distinguant, le tracé (téléologique) du courant à la fonction (éternelle) de résorption féconde. Elle aime la figure cyclonique, issue d'une poétique du «souffle» analysée par Hart (2004: 141). Ponctuels et imprévus, nés au large océanique, ses éléments cycliques et directionnels sont fusionnés en une spirale défiant tout essentialisme.

En effet, dans La Commune de Michel, le regard rétrospectif sur Paris - assiégé malgré le rempart naturel qu'est la Seine - se mue en évocation d'exil. La phrase initiale des mémoires traite de politique: «Dans la nuit d'épouvante qui depuis décembre couvrait le second Empire, la France semblait morte; mais aux époques où les nations dorment comme en des sépulcres, la vie en silence grandit et ramifie" (19). L'Histoire cède vite à la métaphysique dans la prose figurée qui suit:

[L]es événements s'appellent, se répondent pareils à des échos.

[...] Là-bas, par les cyclones, quand le ciel et la terre sont une seule nuit, où râlent comme des poitrines humaines les flots lançant, furieuses [sic], aux rochers leurs griffes blanches d'écume, sous les hurlements du vent, on se sent vivre au fond des temps [...].

Par les tourmentes révolutionnaires au contraire l'attirance est en avant (Commune: 19).

Ce violent lyrisme, s'il brouille le repère chronologique au profit de la spatialité, opère plus qu'une fusion stratigraphique entre différents lieux: il tente de réconcilier le constat raisonné de l'échec politique avec la foi militante. Pour la figure d'exception qu'est Michel, le défi est justement celui de survivre à l'exil: contrairement à Lissagaray et Vallès qui fuient en Angleterre, elle se livre aux Versaillais en échange de la liberté de sa mère, capturée en guise d'appât (Commune: 258).

Le fait d'avoir survécu pèse lourdement sur Michel. Dans cette optique, il est utile de comparer son destin à celui de Gustave Flourens (Bruhat et al. : 234-242). Il est savant et lettré, professeur au Collège de France et fils du célèbre physiologiste Pierre Flourens. Figure romantique, il se bat en Crète contre l'Empire Ottoman vers 1866 et retourne en France poursuivre ses activités révolutionnaires. Il questionne très tôt la stratégie de Trochu, qui ne permet à la milice de se joindre à l'armée qu'en petits contingents qui la fractionnent (Shafer 2005: 77). Le 3 avril, Flourens tente de faire reculer les Versaillais, qui ont Chatillon dans leur poussée pour écraser la Commune (tout juste proclamée). L'heure est grave mais Flourens ne veut pas replier, comme l'écrit Cipriani, son fidèle aide de camp, cité par Michel: "'À ma demande de nous mettre en route il refuse et, descendant de cheval, il se mit à marcher sur le bord de la rivière" "(Commune: 165). Lissagaray aussi décrit la scène: "Las et découragé, Flourens se coucha sur la berge et s'endormit. Cipriani avisa une maisonnette voisine près du pont [...], fit prix d'une chambre où Flourens le suivit» (2000: 182). À Rueil, entre les deux boucles de la Seine, on le dénonce. Ce qui suit grave à jamais le fleuve dans le mémorial communard:

Flourens $[\ldots]$ est conduit sur le bord de la Seine [...], tête nue, bras croisés. Un capitaine de gendarmerie, Desmarests, accourt à cheval, hurle: «C'est vous, Flourens, qui tirez sur mes gendarmes!» et se dressant sur les étriers, lui fend le crâne d'un coup de sabre si furieux qu'il lui fit deux épaulettes, dit un gendarme qui avait le mot jovial (Lissagaray, Histoire de la Commune: 186).

L'opiniâtreté de Flourens est frappante: il défie même les conseils de son ami. Son geste solitaire est provoqué par une attaque sur Neuilly, qui longe la rive droite au nord-ouest de Paris. Lors des deux mois qui séparent le début de la Commune de la Semaine Sanglante, seuls les plus dévoués livrent ce combat qui éclate en une gerbe de sorties réactives (pour ne pas dire suicidaires) contre l'ennemi. 
Le garibaldien connaît un sort bien diffèrent de celui de l'institutrice qui, devant le Conseil de guerre, s'exclame: "J'ai fini! - Si vous n'êtes pas des lâches, tuez-moi» (Commune: 294). (Le pouvoir se gardera bien d'en faire une martyre.) La mort du savant alimentera les légendes et inspirera le bataillon des "vengeurs de Flourens" qui, aux derniers jours de la Commune, escortera à sa mort Vaysset, embauché par Versailles pour soudoyer le fédéré Dombrowski qui résiste à ce piège (Merriman 180; Commune: 236). La fusillade du traître, près de l'île de la Cité, prend sa place dans le mémorial de 1871, tout comme l'image du héros mort sur la berge. Vallès inclut dans L'Insurgé ce "plongeon dans la Seine», "du haut du Pont-Neuf» (351). Cette mort fait écho à celle du mouchard Vincenzini, noyé près du canal Saint-Martin selon le Journal des commissaires de police de 1874 (180).

Michel est pourtant très active lors de la défense contre Versailles. La Commune rapporte qu'elle se bat précisément là où les généraux Vinoy et Ducrot avaient mal agencé leur combat contre Bismarck (soit dans la boucle fluviale sur le front méridional de la capitale). Ce renversement illustre la sévérité de la déterritorialisation subie en 1870-71. L'insurgée qui rédige ses mémoires connaît la suite des choses, ce qui confère une qualité irréelle à ses souvenirs. Il convient d'ailleurs de citer l'aveu qui les précède:

J'avais écrit d'abord ce volume sans rien raconter de moi; sur l'observation de mes amis, j'ai ajouté quelques épisodes personnels aux premiers chapitres, malgré l'ennui que j'en éprouvais; puis il s'est produit un effet tout opposé: en avançant dans le récit, j’ai aimé à revivre ce temps [...] qui fut ma véritable existence, et j'aime aujourd'hui à l'y laisser mêlée. [J]e regarde au fond de ma pensée comme en une suite de tableaux où passent ensemble des milliers d'existences disparues à jamais (Commune: 168-169).

Cette insolite captatio benevolentiae illustre un aspect frappant de la prose michélienne, qui mêle la transparence à l'extravagance et la tendresse à l'entrain. Consciente de son sort singulier, elle transmet des vignettes dont la poétique inédite occulte parfois les allusions aux lieux parcourus. Michel, marchant avec le $61^{\mathrm{e}}$ bataillon, débute au Champ-de-Mars aux abords de la Seine, séjourne à Issy; elle va «à Montrouge avec La Cecilia, ensuite à Neuilly avec Dombrowsky" (Commune: 169-173). L'insurgée les suit donc dans des espaces fédérés défendus avec acharnement. En effet, ces positions, situées de part et d'autre de la porte de Saint-Cloud, seraient pour la force versaillaise un point d'entrée stratégique dans Paris. Ces lieux, revus à l'ombre d'une défaite précipitée justement par une telle percée, ne peuvent que prendre une dimension spectrale. Les tableaux issus du "fond de [l]a pensée» de Louise font voir son style. Aux tranchées de Clamart, elle se retrouve avec un "ancien zouave pontifical converti à la Commune», «d'un noir de jais, avec des dents blanches pointues comme celles des fauves; il est très bon, très intelligent et très brave» (Commune: 170). Elle rapporte leur conversation:

- Quel effet vous fait la vie que nous menons?

- Mais, dis-je, l'effet de voir devant nous une rive à laquelle il faut atteindre.

- Moi, reprit-il, ça me fait l'effet de lire un livre avec des images (Commune: 170).

Ce curieux passage fait écho à l'exotisme de l'exil calédonien et à l'idéal sublimé. En même temps, il suggère tout ce qui sépare l'imaginaire michélien du tableau quasi-allégorique peint par Cipriani à Montretout. Ici la tête du soldat noir n'est plus au sol, mais remplie d'images créant une complicité avec l'institutrice qui adapte hardiment le savoir de son temps à ses convictions. En témoigne son avis sur les œuvres d'art trouvées dans un couvent jésuite près d'Issy, où le bataillon passe la nuit: 
Les tableaux qui ornent les murs ne valent pas deux sous, à part un portrait qui donne bien l'idée d'un caractère, il ressemble à Méphistophélès, ce doit être quelque directeur des jésuites; il y a aussi une adoration des Mages dont l'un ressemble, en laid, à notre fédéré noir, des tableaux de chronologie sainte et autres bêtises (Commune: 170).

Puis, rendue à la barricade du Nord, longeant la boucle fluviale de Neuilly, "près de la maison où [reste] Dombrowski avec son état-major», elle décrit «des déluges d'artillerie versaillaise»: «on eût dit [...] qu’un océan se versait du ciel» (Commune: 192). Encore une fois, le danger se mêle au cocasse:

Une nuit que les camarades avaient voulu que j'allasse me reposer, je vis près de la barricade une église protestante abandonnée dont l'orgue n'avait que deux ou trois notes cassées; j'étais en train de m'y amuser de tout mon cœur quand apparut [...] un capitaine de fédérés avec trois ou quatre hommes furieux. [...] Ainsi finit mon essai d'harmonie imitative de la danse des bombes (Commune: 192).

De Neuilly à Clamart, du nord au sud des hauts de la Seine - et donc de part et d'autre du lieu fatidique qui livrera la Cité à Thiers -, Michel défie foi et raison en naviguant entre rage en humour.

Il ne faudrait pas prendre l'apparente frivolité de l'organiste pour de l'insouciance. Au contraire, sa dissipation semble être largement tactique et la servira bien lors de contacts dangereux avec les autorités (Commune: 76-77, 146-47, 262). D'ailleurs, celle qui croyait n'être pas un «mauvais soldat» cite à l'appui un passage du Journal officiel de la Commune rapportant que « [d] ans les rangs du $61^{\mathrm{e}}$ bataillon combattait une femme énergique, elle a tué plusieurs gendarmes et gardiens de la paix» (Commune: 168). Insérée dans ce milieu bien masculin, elle affirme:

Lorsque le $61^{\mathrm{e}}$ rentrait pour quelques jours, j'allais avec d'autres, je n'aurais voulu pour rien au monde quitter les compagnies de marche et, depuis le 3 avril jusqu’à la semaine de mai, je ne passai à Paris que deux fois une demi-journée. Ainsi j'eus pour compagnons d'armes les enfants perdus dans les hautes bruyères, les artilleurs à Issy et à Neuilly, [...] ainsi je vis combien furent braves les armées de la Commune, combien mes amis Eudes, Ranvier, La Cecilia, Dombrowski comptèrent leur vie pour peu (Commune: 168).

Si ces notes sont justes, il y a fort à parier que la combattante s'est trouvée à traverser la Seine, sinon à défendre les espaces les plus périlleux aux environs du fleuve. En termes géocritiques, la résistance déployée jusque-là sur un axe transversal cède à une tactique tous azimuts visant à prévenir la percée versaillaise. Westphal y verrait une ultime "multiplication de points focaux», née du désespoir. Ces mêmes territoires se mêlent aux éléments néo-calédoniens de l'imagerie michélienne. Le fleuve-rempart prend le large, happant volontiers ambiances, personnages et quelques drôleries.

Il y a lieu, d'ailleurs, de s'attarder à la poétique polissonne qui semble permettre à Michel de concilier son destin d'exception et son souci de solidarité. Michel admire le savoir des tirailleurs. De La Cecilia et de Dombrowski, elle écrit: «[c]es deux hommes, qui physiquement n'avaient aucune ressemblance, faisaient la même impression pendant une action, le même coup d'œil rapide, la même décision, la même impassibilité» (Commune: 168). Elle n’a toutefois pas la prétention de les imiter, ayant le sens du ridicule et de ses limites. Cette modestie est loin d'être de la docilité. Ses dons singuliers trouvent sans aucun doute leur exutoire dans sa pratique d'institutrice mais lui valent aussi de se soustraire à l'abnégation féminine associée à cette profession - de fait, son immense popularité tient au paradoxe d'un altruisme outrecuidant. 
Assoiffée des savoirs de l'époque, elle sait les agencer à ses fins. Aux démunis qui, au gré d'infortunes et d'injures, en viennent à craindre le changement et l'ouverture, elle veut inculquer une méfiance envers le tribalisme, qu'il soit militaire ou conventuel. À ce titre, sa prose - album d'images savamment criard, ralliant enfants perdus, zouaves et rivages mystérieux - s'avère subversive. Cela explique qu'elle soit admise chez les plus célèbres communards dont elle s'accommode des bravades. Son œuvre recense des comportements abusifs chez les dirigeants. Il va sans dire qu'elle trouve leur conduite passible de représailles communardes. Elle tolère l'erreur mais déteste la mauvaise foi.

Sa narration des scènes du 22 janvier en témoigne. Elle loue "la précision mathématique» de Cipriani qui libère Flourens de la prison de Mazas mais elle s'attarde à décrire la grève (Commune: 95). Trochu y est flanqué de ses "mobiles bretons", force catholique bardée de scapulaires (Lefebvre 1965: 180). De l'hôtel de ville, ils visent la foule «en grande partie désarmée» (Commune: 96):

Debout devant les fenêtres maudites, je ne pouvais détacher mes yeux de ces pâles faces de sauvages, qui sans émotion, d'une action machinale, tiraient sur nous comme ils eussent fait sur une bande de loups et je songeais: Nous vous aurons un jour, brigands, car vous tuez, mais vous croyez; on vous trompe, on ne vous achète pas, il nous faut ceux qui ne se vendent jamais (Commune: 98).

Elle a déjà vu ces hommes le 31 octobre, date où Flourens et Blanqui rendent captifs les dirigeants en demandant le vote municipal. C'est alors l'obéissance des réservistes qui la fascine: «leurs yeux bleus fixés dans le vague, [ils] se demandaient si M. Trochu ne débarrasserait pas bientôt la France des criminels» pour qu'ils puissent «revoir la mer [et les] landes où s'ébattent les poulpiquets» (Commune: 87). Le discours indirect (pressenti dans l'appellation du général) y est révélateur: Michel s’identifie plus volontiers aux exaltés qu'aux bien-pensants; et, le souvenir superposant les deux dates, elle lance aux moblots: «nous vous aurons un jour la liberté»; "c'est vous qui avez fait cela [...] mais vous, du moins, vous êtes des fanatiques et non des vendus (Mémoires 2002: 119).

C'est que le recul amalgame deux moments en un espace: la demande du droit de vote puis ses effets funestes. Louise fait le décompte en voyant tomber, tout près d'elle, une femme «en noir, grande et qui me ressemblait» (Commune: 98) La passivité du 31 octobre anticipe l'agression du 22 janvier et la mystique du large océanique semble se confondre avec la pluie des balles; ce ne serait d'ailleurs pas la première fois que la proscrite assimile la grêle de la mitraille à l'orage d'été, lui-même confondu à l'averse tropicale (Commune: 191-192, 246). Du reste, sa détermination ne doit pas faire oublier que la kyrielle d'esquilles lumineuses qu'elle dépeint trahit son traumatisme. Ces fragments de chocs ravivés s'immisceraient non seulement dans ses souvenirs de combats menés en hauts de Seine, mais aussi, désormais, dans ceux des premières assemblées au cœur de la Cité.

L'attrait du large (qui semble toujours vouloir se substituer au tracé prédéterminé du fleuve) et la pulsion vers l'ailleurs (qui fait sans cesse irruption dans l'évocation de la Cité et de ses environs) s'unissent dans une poétique de déplacement chez Michel. Les lieux qu'elle ressuscite sont investis par un imaginaire tant géographique que politique, né du fait qu'elle survit à la Commune. Comme bien des figures d'exception, elle incarne la nécessité de défier la norme pour faire avancer sa cause. En même temps, un militantisme bien entendu exige de ne pas perdre de vue les réalités ordinaires. Il en résulte une impasse pour celle qui semble trop réaliste pour nier l'échec mais reste trop passionnée pour taire ses principes. En somme, la figure de la spirale semble s'offrir comme trope proprement idéologique à la condition du militantisme lucide. Si la visée du progrès doit composer avec l'éternelle inertie, l'élément directionnel qu'est la ligne peut se rallier à l'infini du cercle; le mouvement se répète mais sur des plans successifs où le déplacement empêche le cloisonnement. Louise Michel refuse aussi 
bien les lendemains chantants que le désespoir. Sous sa plume, le tracé du cyclone intègre la perte de ses camarades tout en questionnant la téléologie du progrès épousée par les républicains bien-pensants - ceux-là mêmes qui, trouvant que les insurgés heurtent le cours des choses, justifient leur violence de modérés. Tout cela se retrouve dans son évocation physique et symbolique des lieux communards découpés par la Seine, principe figuratif fédérant ses souvenirs.

Michel préfère donc un calcul fidéiste à la mystique d'une répression raisonnable. Aussi s'émerveille-t-elle devant les prouesses généreuses et les savoirs désintéressés. Elle les inclut dans ses réflexions, où la science a une place de choix. Pour elle, Montretout est la tombe de Gustave Lambert, «qui, peu de temps avant la guerre, organisait une expédition pour le pôle Nord " (Commune: 93). À Clamart, elle lit Baudelaire avec un étudiant désirant calculer des probabilités de tirs d'obus, étrange projet qui manque de les faire tuer (Commune: 171). Elle décrie l'arithmétique des vainqueurs qui inscrivent au passif la mort du général Lecomte - lui qui avait tenté de prendre les canons de la milice. (Il est exécuté quand, dans la colère, "les fusils partent d'eux-mêmes») (Commune: 130). Valent tout autant, aux yeux de Louise, le meurtre de civils manifestant et les morts de Courbevoie, où les fédérés trouvent «rangés sur le quai les cadavres des prisonniers» de Versailles, alors qu'un décret est émis sur la protection des otages (Commune: 153). Elle s'indigne qu'Élisée Reclus soit "expédié aux pontons dans des wagons à bestiaux", transport de cruauté notoire (Commune: 159) : si les soldats y "entendaient quelque bruit», ils "avaient l'ordre [de] décharger leur revolver par les trous pratiqués à cause de l'air (l’ordre fut exécuté). [...] Le sang ne séchait pas [sur] les pavés, la terre gorgée n’en pouvait plus boire, on croyait encore le voir ruisseler pourpré sur la Seine»(Commune: 250).

\section{Seine et savoir: ressacs de l'idéologie michélienne}

Dans la Cité michélienne, le geste gratuit trouve sa plus haute expression dans la crosse levée de ceux qui refuseront de reprendre les canons. Posé dans l'espace autarcique des Buttes, il ramène la démocratie au cœur de Paris, en fédérant les moyens. Ce sacrifice libère l'énergie et le talent technique, bientôt ralliés sur la grève de l'hôtel de ville. Michel rêve en effet de science citoyenne. À preuve, un petit passage où le viril optimisme d'un Verne ou d'un Figuier cède à l'esquif et l'engin exhibés avec fantaisie: "dans la grande paix de l'humanité, la terre sera connue, la science familière à tous, [les] flottes traverseront l'air et glisseront sous les flots, parmi les coraux" (Commune: 93). Le ton bon enfant, d'un entrain un peu suspect, fleure la savante dissolution de Louise. Celle-ci contraste avec l'horreur de mai 1871, où le secret monnayé triomphe sur le savoir-faire généreux. Un informateur, Ducatel, «ancien officier d'infanterie de marine", "traître encore sans emploi», alerte l'armée versaillaise que la porte de Saint-Cloud est mal gardée (Commune: 225). Lefebvre précise la cause: fête musicale avec levée de fonds pour orphelins (1965: 426). Ce détail transforme la Seine qui, de rempart, devient le conduit, entre feu et eau, par lequel les canonnières abattront la Commune.

Cette chasse évoque l'ultime déterritorialisation du conflit civil car la canonnière, arsenal lourd, est vouée au combat frontalier marin. Abandonnée lors d'un repli, elle tient du trophée. Or, Lissagaray peint ainsi la fuite du 18 mars: "Vinoy emmenait à Versailles régiments, artillerie, bagages. Les soldats se traînaient, insultaient les gendarmes. L'état-major [avait] perdu la tête, oubliait dans Paris [toutes] les canonnières qu'il eût suffi d'abandonner au cours de l'eau» (2000: 119). Michel se borne au détail: «Vinoy s'enfuit de la place Pigalle laissant, disait-on, son chapeau» (Commune: 130).

Solidaire jusque dans son usage de la rumeur, l'auteure comprime aussi la scène de mort: «Bientôt de la caserne Lobau le sang en deux ruisseaux s'en alla vers la Seine: longtemps il y coula rouge» 
(Commune: 240). L'image convenue du sang versé est à la mémoire des camarades; mais elle lui permet aussi d'éviter les factions qui surgiront chez les survivants. Lissagaray, avec le recul, déplorera le fait qu'au 8 février, « $[\mathrm{t}]$ el fut élu pour avoir inventé une canonnière, tel autre comme mystique» (82-83). De même, l'adversaire qu'est Maxime Du Camp traite les marins communards de "gens pris à l'aventure", "garçons de lavoir", "rôdeurs de rivières" et "monomanes de canotage" (Les Convulsions de Paris: 29-30); or, il laisse voir malgré lui la valeur du savoir manquant aux Versaillais qui, le 28, veulent suivre les fédérés. Pour «manœuvrer les canonnières, ce n'étaient ni les officiers ni les marins qui manquaient, c'étaient les mécaniciens»; Du Camp s'amuse alors d'une "fable» contée par les hommes de Thiers au sujet des insurgés afin de trouver de tels ouvriers (Les Convulsions de Paris: 101).

Michel, quant à elle, se révèle sévère envers des savoirs qu'elle estime autrement vulgaires. Avec une verve puérile, elle les met en vignette au tout début de son chapitre sur le 4 septembre:

Les anciens braillards «À Berlin», tout en soutenant encore que l'armée française était partout victorieuse, laissaient échapper de lâches tendances vers la reddition, qu’on leur faisait rentrer dans la gorge, en disant que Paris mourrait plutôt que de se rendre, et qu’on jetterait à la Seine ceux qui en répandraient l'idée; ils allaient ramper ailleurs (Commune: 69).

Au couvre-chef de Vinoy, elle préfère les «chapeaux empanachés» des francs-tireurs garibaldiens que méprise Versailles (Guérin 1966: 38). Sa Cité fond les hauts de Seine dans les océans d'ailleurs, butins de l'exil. Ses cloaques abritent des fraternités insolites: «Ceux qui aimaient à rire» (comme si elle n'en était pas) disaient que "quelques agents de Versailles, envoyés par M. Thiers», «devaient s'introduire par des bouches d'égout, mais ils avaient si mal calculé leur affaire» que, pris «à l'orifice, ils durent appeler des ennemis de bonne volonté» (Commune: 193). Ce fleuve turbulent vit pourtant une accalmie en avril 1871. Pendant deux semaines, sa Commune est le sanctuaire de «M. Bourdouze, chimiste» et de son «appareil électrique» qui " télégraphiait sans fils», car l' "Académie des sciences l'avait autorisé à faire des expériences entre les ponts sur la Seine» (Commune: 155). Clapotements dans le fleuve du Progrès, «les savants s'occupèrent de tout dans une paix profonde» (Commune: 155).

\section{Références bibliographiques}

Azéma, J.-P. et M. Winock. 1970. Les Communards. Paris: Seuil.

Bruhat, H. et al. 1970. La Commune de 1871. Paris: Éditions sociales.

Dansette, A. 1944. Les Origines de la Commune de 1871. Paris: Plon.

Du Camp, M. 1881. Les Convulsions de Paris: les sauvetages pendant la Commune. Troisième tome. Paris: Hachette. Eichner, C. J. 2004. Women in the Paris Commune. Bloomington: Indiana University Press.

Forrest, A. 2006. "La mémoire de la Garde nationale "révolutionnaire" au XIx siècle». Dans La Garde nationale entre nation et peuple en armes, sous la direction de S. Bianchi et R. Dupuy. Rennes: Presses Universitaires de Rennes: 503-516. DOI: 10,400 0/books.pur .16645.

Guérin, A. 1965. 1871 la Commune. Paris: Hachette.

Gullickson, G. L. 1996. Unruly Women of Paris: Images of the Commune. Ithaca: Cornell University Press.

Hart, K. 2004. Revolution and Autobiography in Nineteenth-Century France. Amsterdam: Rodopi.

1874. Journal des commissaires de police: recueil mensuel de jurisprudence, de législation et de doctrine 20. ark:/12148/ bpt6k54591704. Consulté le 15 juillet 2018.

Larrère, M. 2007. «La garde nationale: une milice de citoyens armés». Dans L'Histoire. Colonisation: les massacres oubliés 318. URL: http://www.lhistoire.fr/la-garde-nationale-une-milice-de-citoyens-arm\%C3\%A9s.

Lefebvre, H. 1965. La Proclamation de la Commune. Paris: Gallimard. 
9 décembre 1870. Le Figaro. "Chronique de Paris» 343. ark: /12148/bpt6k272068q. Consulté le 17 juillet 2018.

Lissagaray, P.-O. 2000. Histoire de la Commune de 1871. Paris: La Découverte. [1896].

Marx, K. 1963. La Guerre civile en France 1871 (La Commune de Paris). Paris: Éditions sociales.

Merriman, J.M. 2014. Massacre: The Life and Death of the Paris Commune of 1871. New Haven: Yale University Press.

Michel, L. 1999. La Commune: histoire et souvenirs. Paris: La Découverte. [1898].

Michel, L. 2002. Mémoires. Paris: La Découverte. [1886].

Michelet, J. 1888. Histoire de la Révolution française. Tome I. Paris: Lemerre.

Montel, N. 2001. "Chronique d'une mort non annoncée: l'annexion par Paris de sa banlieue en 1860 ", Recherches contemporaines (Université Paris X) 6:217-254. https://hal.archives-ouvertes.fr/hal-01264883/ document.

Montel, N. 2012. "L'agrandissement de Paris en 1860: un projet controversé», dans Agrandir Paris (18601970), sous la direction d'A. Fourcault et F. Bourillon. Paris: Publications de la Sorbonne: 99-111. DOI: 10,400 0/books.psorbonne.2393.

Note sur le concours apporté par la marine pour la répression de l'insurrection de Paris. Paris: Dupont, 1871. https:// gallica.bnf.fr/ark:/12148/bpt6k5447308k?rk=21459; 2. Consulté le 15 juillet 2018.

Priollaud, N. 1983. 1871: La Commune de Paris. Paris : Liana Levi.

Roubaud, N. 1954. Rochefort intime. Paris: Nouvelles éditions latines.

Rougerie, J. 2014. La Commune de 1871. Paris: Presses Universitaires de France. [1988].

Shafer, D. A. 2005. The Paris Commune. Houndsmills: Palgrave.

Troche, N.-M. 1864. Notice historique sur l'ancienne commune de Belleville annexée à Paris et sur sa nouvelle église. Paris: Juteau.

Vachon, M. 1880. Le Château de Saint-Cloud, son incendie en 1870. Paris: Quantin.

Vallès, J. 1923. L’Insurgée. Paris: Fasquelle. [1886].

Viviani, R. 1901. Histoire socialiste. 7: sous la Restauration, sous la direction de J. Jaurès. Paris: Rouff.

Westphal, B. 2000. "Pour une approche géocritique des textes». Dans La Géocritique mode d'emploi, sous la direction de B. Westphal. Limoges: PULIM: 9-39.

Westphal, B. 2007. La Géocritique: réel, fiction, espace. Paris: Minuit. 